\title{
A coherentist conception of ad hoc hypotheses
}

\author{
Samuel Schindler \\ sks@css.au.dk \\ forthcoming in Studies in History and Philosophy of Science
}

\begin{abstract}
What does it mean for a hypothesis to be ad hoc? One prominent account has it that ad hoc hypotheses have no independent empirical support. Others have viewed ad hoc judgements as subjective. Here I critically review both of these views and defend my own Coherentist Conception of Ad hocness by working out its conceptual and descriptive attractions.
\end{abstract}

Keywords: ad hoc; confirmation; independent support; novel success; coherence

\section{Introduction}

It is widely agreed-amongst scientists and philosophers alike-that a good hypothesis ought not to be ad hoc. Consequently, a theory that requires ad hoc hypotheses in order to overcome empirical tests ought to be less acceptable for a rational agent than a theory that does without (or with fewer) ad hoc amendments. But what precisely does it mean for a hypothesis to be ad hoc? This is what this paper is about.

Concept clarification is one of the central tasks of philosophy. Usually, it involves an explicandum and an explicatum, viz. a pre-analytic concept that is to be explicated and a concept which explicates this concept, respectively (Carnap 1950). For example, the concept TEMPERATURE explicates our preanalytic concepts of WARM and COLD, and allows us to be much more precise and clear in the way we communicate with each other. In the case of ad hocness, the pre-analytic concept is something along the lines of "was introduced for the sole purpose of 'saving' a theory which faces observational anomalies". Whilst this hunch may suffice for all practical purposes, it only tells us something about why ad hoc hypotheses are used; it tells us about 
motivations. What we should want as philosophers, however, is an account of ad hocness that can tell us something about why ad hoc hypotheses ought to be avoided in theory choice. In other words, it should tell us why ad hoc hypotheses are epistemically deficient.

It goes without saying that a good account of ad hoc judgements in science must take into consideration how scientists actually use ad hoc judgements. Traditionally, discussions of ad hocness have been informed by historical case studies. The present paper will follow this tradition. There might be other ways of investigating the meaning of ad hocness by descriptive means (interviews with living scientists?), but the history of science offers an established way of seeking descriptive accuracy of philosophical accounts. At the same time, pure historical description of ad hoc judgements as made by scientists will not suffice either. Scientists generally do not explicate what grounds their judgements; they just make them. Thus, some substantive philosophical work is indispensable in the project of determining the meaning of ad hocness by descriptive means.

There are important ramifications of the project of explicating the meaning of ad hocness. First, it affects philosophical theories of theoryconfirmation, since it will tell us about the reasons for why theories that are amended by ad hoc hypotheses should receive less confirmation than theories that are not so amended. Since, second, the realism debate proceeds on the basis of a certain view of theory-confirmation, an explication of ad hocness has the potential for influencing to what kinds of theories realists commit. Although the recent realism debate has focused on novel success as a necessary condition for realist commitment, non-ad hocness is recognized as equally important by some commentators (Psillos 1999). Others even see the rationale of novel success deeply tied to the notion of ad hocness: "it is wrong to regard the downgrading of ad hoc explanations and the apparent upgrading of genuine predictions as two separate methodological phenomena - they are at root the same phenomena" (Worrall 1989, 148).

Philosophers of science were once very interested in specifying the epistemic meaning of ad hocness; mostly in the 1960-1980s. The currently perhaps by far most popular epistemic account of ad hocness is the view that associates ad hocness with the lack of independent support. According to that 
view, a hypothesis is ad hoc when it has no empirical support other than the evidence it was invoked for. However, not everybody agrees that ad hocness is an epistemic concern in the first place. Instead, some have viewed judgements about a hypothesis being ad hoc as subjective aesthetic judgements with no relevance to theory confirmation.

This paper is structured as follows. In Section 2, I critically review two prominent explications of ad hocness, namely independent testability and independent support. In Section 3 I introduce subjectivist accounts of ad hocness. In Section 4 I propose and advocate my own account of ad hocness, the Coherentist Conception, by working out its conceptual and descriptive attractions. In Section 5 I argue in detail that the history of one of the most pragmatic ad hoc hypotheses, namely the Lorentz-Fitzgerald contraction hypothesis, supports my account. I conclude this paper in Section 6.

\section{Extant accounts of ad hocness}

Two popular ways of explicating the meaning of ad hocness is in terms of independent testability and independent support. In this section, I will discuss these two proposals in turn.

\subsection{Ad hocness as lack of independent testability}

The philosopher with arguably the biggest impact outside the discipline, Karl Popper, was very much driven by the attempt to formulate conditions that would disqualify as unscientific the ad hoc maneuvers of psychoanalysis and other alleged pseudo-sciences, and was once even described as "introducing new, non-justificationist criteria for appraising scientific theories based on antiad hocness." Lakatos (cf. Popper 1959a, 42, 1978 39). Popper believed that ad hoc hypotheses would decrease the falsifiability of the theory they were introduced to save (Popper 1959a, 82f.) and that "degrees of ad hocness are related (inversely) to degrees of testability and significance" (Popper 1959b). If a hypothesis which is added to a theory entails only the evidence that is troublesome to this theory, then the falsifiability of the theory is decreased: there is one state of affairs less that could threaten the theory in question. On the other hand, the falsifiability of the theory is increased when the added hypothesis makes new predictions. This is why Popper also claimed that ad hoc hypotheses "cannot be tested independently" $(1974,986)$, i.e., they cannot be 
tested other than on the basis of the evidence they were introduced to account for. At least this is the idea. Barnes (2008, 11), following Bamford (1993, 349-50) has -I think rightly-questioned Popper's alleged connection between ad hoc hypotheses and decreased testability. For example, it is for example not plausible that the hypothesis "bread nourishes" when amended to "all bread nourishes except that grown in a particular region of France" after the relevant discovery, would result in a decrease in testability. Independent testability also seems not the right way of thinking about one of the most emblematic ad hoc hypotheses, namely the Lorentz-FitzGerald contraction hypothesis (LFC), famously introduced to save the aether theory from refutation in the face of the famous Michelson and Morley aether drift null result.

There is strong evidence that contemporary scientists regarded the LFC as an ad hoc hypothesis. Einstein, for instance, wrote about LFC that "[t]his manner of theoretically trying to do justice to experiments with negative results through ad hoc contrived hypotheses is highly unsatisfactory" (Einstein in Warburg 1915, 707). To Michelson, who was initially actually quite keen to explain away his null result in favor of the ether theory felt that "such a hypothesis seems rather artificial" (cited in Holton 1969, 139). Even Lorentz, who had developed the LFC, admitted that it first appeared 'far-fetched' and that it depended on an assumption (namely that the same laws apply to intermolecular forces that apply to electrical ones) which 'there is no reason to make' (Lorentz 1895 in Einstein et al. 1952, 6). In response to criticism by Poincare in 1900, Lorentz also admitted that "[surely] this course of inventing special hypotheses for each new experimental result is somewhat artificial" (Lorentz 1904, ibid., 12-13).

Although Popper in his Logic of Scientific Discovery mentioned the LFC as the only example for his claim that ad hoc hypotheses are not independently testable, Grünbaum (1959) pointed out that LFC actually did entail an independently testable prediction. More specifically, it entailed a positive aether drift result that was different from the positive result implied by the unmodified aether theory. There even was an experiment, namely the so-called Kennedy-Thorndike experiment, which falsified this consequence of the LFC. ${ }^{1}$

\footnotetext{
1 The LFC had it that matter moving through the ether contracts in the direction of motion by the same laws governing the contraction of interference patterns. Whereas the Michelson-
} 
In response, Popper conceded that the LFC could not be disqualified as ad hoc on the basis of it not being independently testable. ${ }^{2}$ The conclusion Grünbaum (1976) drew was this: ad hoc hypotheses can have independent consequences, but when they do, these consequences have no support (because no relevant evidence has yet been gathered), or they have been shown to be false at the time at which the hypothesis is judged ad hoc.

\subsection{Ad hocness as lack of independent support}

The idea that a hypothesis needs independent support for it to not be judged ad hoc is extremely popular amongst philosophers (e.g. Zahar 1973, Schaffner 1974, Leplin 1975, Lakatos 1978, Scerri and Worrall 2001, Worrall 2002, Sober 2008). ${ }^{3}$ However, there is again historical evidence which speaks against such a view. With regards to our example of the contraction hypothesis, the historian Holton remarks that the independent predictions of the LFC were "not of real interest in any case; they were not urged as tests that would decide on its acceptability" (Holton 1969, 177).

\footnotetext{
Morley experiment showed that the speed of light is independent of the orientation of the experimental apparatus, the Kennedy-Thorndike experiment showed that it was independent also of the velocity of the apparatus. For more details see e.g. (Janssen 2002b).

2 Popper nevertheless held onto the independent testability account and claimed that the LFC was at least less independently testable (and therefore more ad hoc) than Einstein's special theory of relativity (which he considered at hoc to some degree too) (Popper 1959b). ${ }^{3}$ A very influential account of ad hocness which embraces the "no independent support" condition is Leplin's (1975). Apart from this condition, Leplin also (amongst a dozen necessary and jointly sufficient conditions) argues for the so-called "non-fundamentality condtion", according to which "there are problems other than [anomalous phenomenon] E confronting [theory] T which there is good reason to hold are connected with E [in certain ways]". This makes it impossible to apply Leplin's account to cases in which (at a particular point in time) there are no other problems or anomalies than E. But surely, a good account of ad hocness should allow for such cases. Also, there seems little reason to suppose a priori (as Leplin does) that every time a theory is considered ad hoc, the anomalous phenomenon can in fact be connected with other phenomena (by a more unified theory). This may be so in some cases, but it shouldn't be presumed for all cases. As another influential account of ad hocness that embraced the no independent support view of ad hocness, we should here also mention Lakatos's (1978), which distinguished between three senses of ad hoc hypotheses (with regards to a research programme): ad hoc1 $=$ the hypothesis makes no new predictions; $\mathrm{ad} \mathrm{hoc}_{2}=$ the predictions a made by a hypothesis are not confirmed; ad hoc 3 = the hypothesis does not is "not form an integral part of the positive heuristics" (fn 1 on p. 112). The first and the second sense are covered by my discussion in this and the previous section. A discussion of the third sense would also lead us too far astray.
} 
Against the independent support idea of ad hocness, one may also appeal to an argument by Barnes (2005) made against Scerri and Worrall (2001) in the context of predictivism (the thesis that novel success counts more than accommodative success). Suppose with Worrall that creationists, when presented with fossils seemingly undermining the creationist doctrine, might respond with a 'Gosse dodge': fossils are just God's playful writings in stone, possibly testing our faith. With Worrall one might think that the Gosse dodge is ad hoc because it has no independent support. However, suppose we view the Gosse dodge as a general hypothesis about all fossils. Then the Gosse dodge would not only be about the particular fossils for which the Gosse dodge has been invented, but also about other fossils throughout the world and fossils yet to be discovered. Arguably, those other fossils, once discovered, would be independent support for the Gosse dodge. Yet the Gosse dodge is clearly ad hoc, regardless (807). One may be unimpressed with this toy example; we will consider a more realistic example in a moment. ${ }^{4}$

Proponents of the independent support view of ad hocness have repeatedly appealed to the discovery of the planet Neptune to make their case (Worrall 2002). In order to accommodate a discrepancy in the orbit of Uranus, Adams and Le Verrier, independently from each other, postulated another planet in the vicinity of Uranus in 1845-46. Neptune was subsequently discovered in 1846. Proponents of the independent support view of ad hocness have taken this to confirm their view (Worrall 2002): the Neptune hypothesis was introduced ad hoc in order to save Newtonian mechanics from refutation, but was rendered non-ad hoc when it received independent confirmation via the discovery of Neptune. However, as Leplin (1982) has pointed out, there is no evidence whatsoever that the scientific community at the time regarded the

\footnotetext{
${ }^{4}$ One might for example object to this example on the basis of (i) the vagueness of the Gosse dodge and (ii) the insufficiency of independence of the support from other fossils. As to the first point, one may criticise that the Gosse dodge is not really falsifiable and therefore should not be taken seriously as a scientific hypothesis in the first place. As to the second point, one may demand on behalf of the proponents of the independent support account, that empirical support must be qualitatively significantly different for it to be independent. I sympathize with both points. Yet I do share the crucial intuition that independent support for a hypothesis is not sufficient for rendering it non-ad hoc.
} 
postulation of Neptune ad hoc, or somehow methodologically unsound. ${ }^{5}$ But if the postulation and discovery of Neptune really is no example for an ad hoc hypothesis, then it cannot lend credence to the independent support condition of ad hocness, or to any account of ad hocness for that matter. ${ }^{6}$ Moreover, it can then also not be the case that introducing a hypothesis to save a theory from refutation is sufficient for that hypothesis to be ad hoc. Something else is needed.

The independent support view of ad hocness is very persistent. In the most recent contribution on the topic of ad hocness, Friederich et al. (2014) discuss several judgments by physicists (and some philosophers) about the Higgs mechanism (HM) of the standard model in particle physics being ad hoc. Friederich at el. list the following features of the HM that arguably underlie physicists' ad hoc judgments: (i) the HM leads to a large number of free parameters for the particle masses that are not determined in a principled fashion but need to be put in 'by hand' on the basis of experimental results, (ii) there are no known fundamental scalar particles in physics apart from the Higgs boson, (iii) the symmetry breaking of the HM, contrary to all other known cases of symmetry breaking, is implemented not dynamically but by fiat, ${ }^{7}$ and (iv) the fact that the bare mass of the Higgs particle is 'fine-tuned' to its interaction mass in an "unnatural way" (by 34 orders of magnitude) is unexplained. ${ }^{8}$ This is known as the 'fine tuning' or 'naturalness' problem and, according to Friederich et al, "is widely regarded as the most severe" argument against the "fundamental scalar character" of the Higgs field. ${ }^{9}$ In other words, the HM has

\footnotetext{
${ }^{5}$ See for example Grant (1852, 164-201) and Grosser (1962). Bamford (1996), similarly, notes that "[ $\mathrm{t}]$ here was no theoretical objection, however, to a planet located at some intermediate distance beyond Uranus" (216).

${ }^{6}$ Incidentally, it is interesting to note with Leplin (1975 314, fn. 17) that Lorentz appears to have regarded the question of whether or not the LFC was ad hoc independently of the question of whether or not there ever could be (positive) experimental tests for it (Lorentz 1885 in Einstein et al. 1952, 6). We shall return to this point later in the paper (Section 5).

${ }^{7}$ Symmetry breaking normally is due to composite rather than fundamental fields. Only in the former case can it be dynamical.

8 In quantum field theory any experimentally determined particle mass is understood as the sum of the 'bare' mass and the 'interaction mass', due to the interaction of the particle with vacuum fluctuations.

${ }^{9}$ Friederich et al. mention two further points: that a non-zero Higgs field in a vacuum is conceptually problematic and 'triviality'. The first of these points, Friederich et al. argue, makes a false presupposition: the Higgs field in the vacuum actually is zero. The second concerns the position of the so-called "Landau pole", basically a limit of the standard model to certain energy
} 
a number of arbitrary, theoretically unmotgivated, features. Interestingly this is also what Weinberg, in his original paper, expressed when he conceded that "[...] our model has too many arbitrary features for these predictions [regarding the electroweak "mixing angle"] ${ }^{10}$ to be taken very seriously" (Weinberg 1967, 1265-66). It is this aspect of arbitrariness that appears to underlie physicists' judgment that HM is ad hoc.

Friederich et al. conclude their analysis by announcing "the end of the ad hoc character of the $[\mathrm{HM}]^{11}$ due to the recent discovery [of the Higgs particle]" (3914). More specifically, they argue that HM is no longer ad hoc, because "the most crucial characteristic of an ad hoc hypothesis", which for them is the lack of independent support, "is no longer obeyed" (3913). Yet at the same time they note that

\footnotetext{
Many physicists [...] now seem to be ready to accept the [HM] as part of physical reality. On the other hand, most of them seem to be not ready to conclude that the [aforementioned] criticisms of the $[\mathrm{HM}]$ were unfounded [...] [since] the experimental confirmation of the $[\mathrm{HM}]$ does not solve any of these problems (3913; original emphasis).
}

But if the reasons for judging the HM ad hoc have not been addressed by the Higgs discovery, then why should we think that the ad hoc status of the HM has changed? The fact that the HM describes a particle that exists is not enough. In the history of science, there have been many ad hoc hypotheses of real entities or phenomena (such as the Planck hypothesis of black body radiation; see 4.1). Yet Friederich et al. seem to implicitly rule out this option, as they go on to suggest only two possible reactions to the Higgs discovery: either supersymmetry theories incorporating the Higgs boson as one amongst several

\footnotetext{
scales. The Landau pole for the standard model depends on the observed Higgs mass. For the currently observed Higgs mass, the Landau pole turns out to be entirely absent. This result, Friederich et al. point out, can be generated only "if the self-coupling of the Higgs boson is assumed to be vanishing, i.e. trivial, while this self-coupling must be non-vanishing for the HM to generate non-vanishing particle masses". For reasons of simplicity, I will leave out those points in my discussion.

${ }^{10}$ The mixing angle of the Weinberg model, which also became known as Weinberg angle, indicates the relative strengths of the neutral and charged weak interactions and the masses of the $\mathrm{Z}$ and $\mathrm{W}$ bosons.

${ }^{11}$ Instead of HM, Friederich et al. use the abbreviation SMHM for Standard Model Higgs Mechanism.
} 
such particles turn out correct, or the HM will no longer be considered ad hoc. ${ }^{12}$ But again, I think this is a false dichotomy: it may well be that HM is and will remain ad hoc despite the discovery of the Higgs particle until a better theoretical device in agreement with the facts has been constructed..$^{13}$ In fact, given that none of the original problems of the HM (which led to the ad hoc judgements) has been alleviated, this seems to be the more accurate picture.

In sum, the independent support view of ad hocness is in trouble. Physicists' view of the HM, the (hypothetical) Gosse dodge, and (I would argue) the LFC show that a hypothesis can be ad hoc even when it does (or if it would) have independent support. Conversely, the Neptune hypothesis-often used as simple illustration for the independent support account-was never deemed ad hoc, even at a time when it had no independent support. The lack of independent support is thus neither necessary nor sufficient for a hypothesis to be considered ad hoc.

\section{Subjectivist accounts of ad hocness}

In contrast to the two accounts discussed in the previous two sections, there are also approaches that deny that ad hocness can be explicated in one particular

\footnotetext{
${ }^{12}$ Friederich et al. offer quotes from three physicists commenting on two of the problems of the $\mathrm{HM}$ as evidence for the second option. But none of these quotes seems to support this view. Friederich et al. refer to a review article on the status of supersymmetry by Feng (2013), a blog entry by Krämer (2013), and a paper on the hierarchy problem by Wetterich (2012). Feng concludes his paper by saying that "weak-scale supersymmetry [motivated by the problem of naturalness, amongst other things] is neither unscathed, nor is it dead", which would seem to suggest that, despite the Higgs discovery, he has not given up the hope that naturalness will eventually be achieved in sypersymmetric theories. There certainly is no evidence that he would regard the HM as less ad hoc than before the Higgs discovery. The same is true of Friederich et al.'s quote of Krämer: Krämer says only that the lack of evidence for supersymmetry in the results of the Large Hardron Collider experiments means that the search for theories incorporating naturalness may have to be given up and not that theories without naturalness, such as the HM, are now fully embraced as non-ad hoc because the Higgs has been found (as Friederich et al. seem to want to suggest). Lastly, it is worth noting that the quote by Wetterich, which Friederich et al. use to argue for a reevaluation of the fine-tuning problem due to the Higgs discovery, is truncated and in fact starts with "It has been shown long ago...", where Wetterich cites an article of his from 1984, i.e., 28 (!) years before the discovery of the Higgs particle.

${ }^{13}$ Physcists seem indeed keen to develop such an alternative. It is for example well known that many physicists were rather disappointed that no further Higgs-like bosons were found at the LHC at CERN, as required by supersymmetry theories. See e.g. Overbye (2012) and Heilprin (2013). String theory research, despite its problems, also still holds the promise of a theoretically more appealing theory than the standard model.
} 
way. Such approaches tend to treat ad hocness as subjective projections or aesthetic judgements.

In a remarkable paper discussing the role of the Michelson-Morley experiment, the historian Holton noted that scientists' judgments about a hypothesis being ad hoc are often accompanied by aesthetic terms such as 'artificial', 'contrived', 'strange', 'surprising', and the like (Holton 1973, 327). ${ }^{14}$ Holton also claims that ad hoc judgments are highly context-dependent, and vary inter- and even intra-subjectively. That is, what might be regarded as ad hoc by some may be seen as non-ad hoc by others, and what might be at regarded as ad hoc one point in time, according to these authors, might be regarded as methodologically sound at a later point in time, even by a single person (Holton 1973, 176-183). In conclusion, Holton urged that philosophical analysis "must be supplemented by an understanding of matters of scientific taste and feeling" (183). Recently Hunt (2012) reaffirms that "scientists' aesthetic sense or 'feeling' governs their judgments in this matter [of ad hocness]" and that the answer to the question of whether a hypothesis is ad hoc is "largely in the eye of the beholder" (13). On the subjectivist account, whether a hypothesis is deemed ad hoc or not (by anyone) has no epistemic bearing. Hunt accordingly concludes that "at the end of the day there seem to be no ad hoc hypotheses and no non-ad hoc hypotheses, only hypotheses - full stop" (Hunt 2012, 13).

I think it is highly questionable that the subjectivist account accurately represents scientists' views of the matter. Contrary to Holton and Hunt I think it is quite obvious that scientists do indeed care about whether or not their hypotheses are ad hoc and that they do view ad hoc hypotheses as epistemically deficient (see e.g. our discussion of the LFC above). Furthermore, if ad hoc hypotheses would be just as good as any other non-ad hoc hypotheses then there would be very little constraint on theorizing other than saving the phenomena. Any theory facing anomalies could be patched up at will without that being objectionable. Since any theory can save any phenomena when

\footnotetext{
${ }^{14}$ Holton even claims that an ad hoc judgment need not be pejorative and that there are "acceptable ad hoc hypotheses". Holton provides no evidence for this claim other than stating that scientists sometimes describe them positively (Holton 1973, 327).
} 
amended ad hoc, how could scientists then rationally deliberate about which theory to adopt?

Contrary to how the subjectivists would have it, the inter-subjective and intra-subjective variance of ad hoc judgements, if it is real, does not entail the conclusion that the subjectivists try to establish. Scientists make mistakes. Thus, when scientists disagree as to whether a hypothesis is ad hoc or not, some might scientists might simply be wrong. And scientists might change their minds about the ad hoc status of a hypothesis for all the wrong reasons. This is of course not to say that none of the reported variance should be taken seriously. On the contrary. What I will argue, though, is that we don't have to go all the way subjectivist and relativist to accommodate such judgments.

\section{A coherentist conception of ad hocness}

Let us start out with another example. The Ptolemaic system of our planetary system famously used the theoretical device of epicycles: the planets were envisaged to move on circles whose centre in turn moved along the circumference of other circles around the central body, believed to be Earth. Although epicycles have become proverbial for ad hoc devices, it was not those to which Copernicus objected when criticizing the Ptolemaic system in his De revolutionibus. In fact, Copernicus himself made heavy use of them (Kuhn 1957). ${ }^{15}$ Instead, what appears to have taken central stage in Copernicus's objections to the Ptolemaic system were concerns about coherence:

[Ptolemaic astronomers have not] been able [...] to discern or deduce the principal thing - namely the shape of the universe and the unchangeable symmetry of its parts. With them it is as though an artist were to gather the hands, feet, head and other members for his images from diverse models, each part excellently drawn, but not related to a single body, and since they in no way match each other, the result would be a monster rather than a man.

(Copernicus cited in Kuhn 1957, 137-8)

I will specify what underlies Copernicus's criticism further in a moment, but I take this to be prima facie evidence for what I call the Coherentist Conception of Ad Hocness (CCAH), according to which a hypothesis $\mathrm{H}$ is ad hoc, iff theory $\mathrm{T}$

\footnotetext{
15 Gingerich (1975) raises doubt that the numbers of epicycles can be determined unequivocally on any of the two systems.
} 
and $\mathrm{H}$ do not cohere or $\mathrm{H}$ does not cohere with the accepted background theories B. ${ }^{16}$ More precisely,

Definition of ad hocness: A hypothesis $\mathrm{H}$, when introduced to save a theory $\mathrm{T}$ from empirical refutation by data $\mathrm{E}$, is ad hoc, iff (i) $\mathrm{E}$ is evidence for $\mathrm{H}$ and (ii) $\mathrm{H}$ appears arbitrary in that $\mathrm{H}$ coheres neither with theory $\mathrm{T}$ nor with background theories B, i.e., neither T nor B provide good reason to believe that $\mathrm{H}$ (possibly specifying a particular value of a variable), rather than non- $\mathrm{H}$ (or some value other than the one specified by $\mathrm{H}$ ).

Condition (i) is trivially true for any ad hoc hypothesis, because ad hoc hypotheses get introduced to accommodate data in the first place. Condition (ii) prominently features coherence relations. Coherence has been described as a measure for "how well things hang together" (BonJour 1985). There is no agreement about what exactly coherence amounts to, but I will assume that if $\mathrm{H}$ coheres with $\mathrm{T}$ or $\mathrm{B}, \mathrm{T}$ or $\mathrm{B}$ will give one good reason to believe that $\mathrm{H}$, rather than non-H. These reasons, by virtue of being provided by $\mathrm{T}$ or $\mathrm{B}$, will be theoretical reasons. ${ }^{17}$ How are theoretical reasons to believe $\mathrm{H}$ to be understood? And when are theoretical reasons good ones?

For $\mathrm{T}$ or $\mathrm{B}$ to provide theoretical reasons to believe that $\mathrm{H}$ (rather than non- $\mathrm{H}$ ), T or B must of course be logically consistent with $\mathrm{H}$. But consistency is not sufficient for $\mathrm{T}$ or $\mathrm{B}$ to provide theoretical reasons to believe that $\mathrm{H}$ (rather than non-H), much as consistency is not sufficient for coherence. ${ }^{18}$ For $\mathrm{T}$ or B to provide theoretical reasons to believe that $\mathrm{H}$, further conditions must be met. In the most straightforward case, $\mathrm{T}$ or $\mathrm{B}$ provide theoretical reasons to believe that $\mathrm{H}$ when $\mathrm{H}$ can be deduced from $\mathrm{T}$ or $\mathrm{B}$. But there are other ways of theoretically

\footnotetext{
${ }^{16}$ McMullin $(1998,133-4)$ was perhaps the first to link ad hocness with the lack of coherence. Yet McMullin's account remains sketchy. He appears to construe coherence in terms of causalunificationist explanation. In his discussion of the Copernican system in astronomy, he remarks about Copernicus that "he is saying that a theory that makes causal sense of a whole series of features of the planetary motions is more likely to be true than one that leaves these features unexplained." (134). See the next section for more details about the unificationist account4.1. ${ }^{17}$ It should be noted that Leplin (1975) lists "H has no independent theoretical support" as one of altogether twelve (necessary and jointly sufficient) conditions for ad hocness, which he spells out as "no laws or principles of other still viable theories are such that their acceptance would constitute good reason for proposing the hypothesis" (11).

${ }^{18}$ In the following, I will, for the sake of simplicity, not write out the contrastive part of "reasons to believe $\mathrm{H}$, rather than non- $H^{\prime \prime}$. Whenever I do use the simplified phrase, I nevertheless do want it to be understood in its complete version. The contrastive part ensures that the reasons provided by $\mathrm{T}$ or $\mathrm{B}$ are relevant reasons to believe that $\mathrm{H}$.
} 
justifying $\mathrm{H}$. For example, a theoretical reason to believe that $\mathrm{H}$ might consist in an explanation for why $\mathrm{H}$ should be the case, by the lights of $\mathrm{T}$ or B. Such an explanation, in turn, could be causal, or it could consist of a subsumption of $\mathrm{H}$ under a regularity. $\mathrm{T}$ or $\mathrm{B}$ might also provide reasons to believe that $\mathrm{H}$ simply in virtue of ruling out scenarios inconsistent with $\mathrm{H}$ (as we shall see in a moment). In other words, I believe it is advisable to adopt a pluralist attitude towards the kinds of permissible theoretical reasons to believe that $\mathrm{H}$, which $\mathrm{T}$ or B can provide, just as one might want to be pluralist about the nature of coherence relations. Lastly, we will demand that for theoretical reasons provided by $\mathrm{T}$ or B to be good ones, $\mathrm{T}$ or B will have to have empirical support. Otherwise, any arbitrarily cooked-up theory could be used to render an ad hoc hypothesis non-ad hoc. At the same time, it should be emphasized that, by the lights of $\mathrm{CCAH}$, good reasons to believe that $\mathrm{H}$ can never be just empirical reasons alone, for they must be provided by $\mathrm{T}$ or $\mathrm{B}$, and thereby be theoretical reasons.

Let us return to Copernicus's criticism of the Ptolemaic system. Copernicus explicated the aforementioned analogy by discussing a number of observations which, on the Ptolemaic system, had to be simply assumed, but for which the Copernican system gave good reasons to believe. ${ }^{19}$ Some of these observations were the maximum elongation of the inferior planets, i.e., the observational fact that Mercury and Venus are never observed beyond a certain angle from the apparent trajectory of the sun on the celestial sphere, i.e., the socalled ecliptic ( $28^{\circ}$ and $47^{\circ}$, respectively). In order to account for this fact, it was decreed in the Ptolemaic system that the centre of the epicycle on which an inner planet would move had to be fixed on a line connecting Sun and Earth. In the Copernican system, in contrast, the inner planets cannot possibly move away from the sun beyond a certain angle, because the inner planets' orbits are encompassed by the Earth's orbit around the sun (see Fig. 1). ${ }^{20}$

\footnotetext{
${ }^{19}$ Another point that was important to Copernicus was that the Ptolemaists had, with the deployment of eccentrics, departed from the Aristotelian principle of uniform motion. For a highly interesting discussion see (Miller 2014).

${ }^{20}$ For a detailed discussion see also (Janssen 2002a).
} 


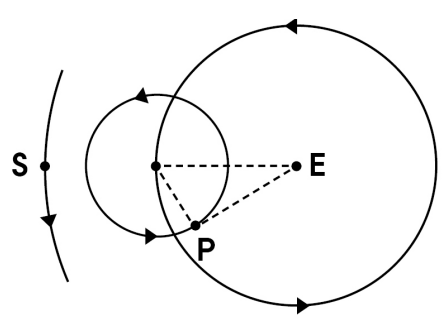

(a)

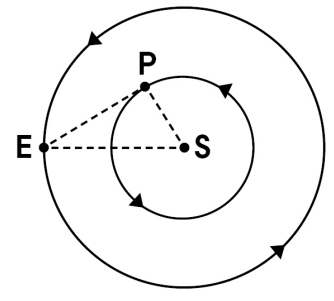

(b)

Fig. 1: The phenomenon of maximum elongation of the inner planets as explained in the Ptolemaic system (left) and the Copernican system (right). From (Kuhn 1957).

The way in which the Ptolemaic system accommodates the phenomenon (E) of maximum elongation is clearly ad hoc, because neither the Ptolemaic system itself, nor the background theories at the time provided any good reasons to believe the hypothesis $H$ that the centre of the inner planets' epicycles must remain fixed on a line connecting the sun and Earth. H was arbitrary, since theoretically unmotivated. In contrast, in the Copernican system it is impossible for the inner planets to move beyond a certain angle away from the sun (as observed from the Earth), as it was surmised $\left(\mathrm{H}^{*}\right)$ that the inner planets' orbits are located inside Earth's. The Copernican system thus provides excellent theoretical reasons to believe that $\mathrm{H}^{*}$, as $\mathrm{H}^{*}$ follows straightforwardly from the Copernican system. ${ }^{21}$

Before illustrating CCAH with further examples, let us clarify and compare CCAH to the extant accounts of ad hocness.

\subsection{Clarifications and comparison with other accounts}

Let us first note that, contrary to a not too uncommon misconception, condition (i) of the CCAH is not sufficient for a hypothesis to be ad hoc. Consider again the stipulation of Neptune after the detection of irregularities in the orbit of Uranus (cf. Section 2). Again, contrary to folklore, there is no evidence that the hypothesis of Neptune was considered ad hoc before Neptune was discovered, even though it was introduced to save Newtonian mechanics. On the CCAH, the Neptune hypothesis was never ad hoc, because Newtonian mechanics did

\footnotetext{
${ }^{21}$ It may be noted that this example is compatible with both the independent testability and independent support notions of ad hocness. I do not claim, however, that these accounts are irreconcilable with all historical cases. But I do claim that my account does better in capturing some of the important cases which extant accounts don't (see Section 2). Thanks to one of the referees of this journal for prompting me to clarify this point.
} 
provide good reasons to believe that there was another planet in the vicinity of Uranus, given the irregularities of its orbit.

My coherentist account of ad hocness is an objectivist account. When a hypothesis does not cohere either with $\mathrm{T}$ or $\mathrm{B}, \mathrm{H}$ will be ad hoc.

Contrapositively, when $\mathrm{H}$ coheres with $\mathrm{T}$ or B, it won't be ad hoc. Yet, the account does allow for a subjective element in ad hoc judgements when it comes to weighting the relevant relations: some scientists might judge some connections of $\mathrm{H}$ to $\mathrm{T}$ or B more important than others. I'm therefore proposing an analogue to Kuhn's model of theory-choice where individuals weigh objective theory properties according to their own (subjective) preferences (Kuhn 1977). Analogously in the case of coherence in my sense, scientists can legitimately differ about which coherence relations they consider to be more important. But that has no bearing on whether or not coherence relations obtain between $\mathrm{H}$ and $\mathrm{T}$ or $\mathrm{B}$. Accordingly, the judgement that some hypothesis $\mathrm{H}$ is ad hoc when as a matter of actual fact $\mathrm{H}$ does cohere with $\mathrm{T}$ or $\mathrm{B}$ would be a false judgements. Subjectivist accounts of ad hocness (Section 3) have no such restrictions. My account thus sets clear limits to the legitimacy of ad hoc judgements.

Relatedly, as we saw, proponents of a subjectivist account of ad hocness have cited degrees of ad hocness as evidence for ad hoc judgements being entirely aesthetic (Section 3). Yet, once again, one can admit the former without drawing the latter (radical) conclusion. CCAH too allows for degrees of ad hocness: the stronger the coherence between $\mathrm{H}$ and $\mathrm{T}$ or $\mathrm{B}$, the less ad hoc $\mathrm{H}$. The strength of coherence may perhaps be gauged both in terms of number and quality of relations. That is, the more and the stronger the theoretical reasons for believing $\mathrm{H}$, as provided by $\mathrm{T}$ or $\mathrm{B}$, the less ad hoc $\mathrm{H}$. It is also worth noticing that degrees of ad hocness allow us to say one theory ought to be preferred over another, regarding ad hocness, even if neither theory manages entirely without ad hoc hypotheses: one theory might just invoke fewer ad hoc hypotheses than the other. In fact, I think one might question whether there is any prominent scientific theory which does entirely without ad hoc assumptions.

Take again the Copernican system: it did not manage to do entirely without ad hoc assumptions. For example, it is a consequence of the Copernican 
system that one should be able to observe a parallax shift, namely an apparent shift in the position of stars, due to the observer's changing positions on Earth moving around the sun. Yet, the effect could not be observed up until the early $19^{\text {th }}$ century due to technological limitations (telescopes were not advanced enough at the time). Copernicus thus had to invoke the idea that the stars were in fact much further away from us than generally thought at the time. However, this hypothesis was incoherent with the accepted background theories of the time and therefore ad hoc. This was just one of the reasons why it took decades before the Copernican system gained wider acceptance (Copernicus also lacked a workable physics for a moving Earth). Regardless, the Copernican system was much less ad hoc, as compared to the Ptolemaic system, when it came to accounting for observations regarding the planets, the sun, and the moon (it, for example, explained in a non-ad hoc fashion the fact that only the planets, but not the sun and the moon, retrogress, and why the frequency of planetary retrogressions decreases from Saturn, Jupiter, and Mars, and increases from Venus to Mercury, and that the superior planets are the brightest in their opposition (Copernicus 1543/1992, 26-7)). Thus, we can say that the Copernican system, when it was first proposed, constituted an improvement with regards to the number of ad hoc assumptions it had to invoke in accounting for many planetary observations, which made it preferable over the Ptolemaic system. At the same time, the Copernican system too had to make do with a number of ad hoc assumptions before it was eventually developed into a system coherent with accepted physics. Thus, the CCAH account, although allowing the basis of comparative theory-choice on the basis of degrees of ad hocness, does not suggest simplified algorithms that do not adequately mirror the complextity of history.

Of course, there are other important considerations in theory choice than ad hocness. Most importantly, a theory needs to be empirically adequate. And if we do not find a theory that is empirically adequate and non-ad hoc, clearly, we should stick to it in any case if it is empirically adequate. I think this is what can be said about the Higgs mechanism after the Higgs discovery, which we discussed in Section 2.2. There is another historical example, which I now want to consider briefly.

Planck famously introduced the concept of quantization of energy into physics in an ad hoc fashion in order to account for black body radiation. He 
himself was not very happy about that and physicists worked for decades to devise a theory that gives us good reasons for the quantization of energy (Kuhn 1987). Thus, although undesirable, ad hoc hypotheses can drive progress not only by way of helping scientists to save the phenomena but also by way of giving incentives to develop theories which render those very same hypotheses non-ad hoc (by providing good reasons for believing in them). Accordingly, the view of norms that recommends itself is not one where methodological norms are categorical, but rather conditional and ceteris paribus. That is, the norm not to devise ad hoc hypotheses has to be weighed against other norms such as "seek hypotheses that are empirically adequate". It can therefore not always be wrong to devise ad hoc hypotheses; it is admissible when no other hypotheses are available. It is nevertheless always desirable to devise hypotheses that are not ad hoc, as the aforementioned example illustrates.

Let us turn to the example of the Higgs mechanism discussed by Friedrich et al. (2014) and recall that they report the following grounds on which it has been deemed ad hoc by physicists: (i) it possesses a large number of free parameters, (ii) there are no other fundamental scalar particles than the Higgs boson, (iii) the symmetry breaking of the Higgs mechanism is different from all other known cases of symmetry breaking, and (iv) the Higgs particle is 'fine tuned' to its interaction mass and unexplained. As we saw in Section 2.2, there is no evidence that the independent support of the Higgs mechanism in the form of the discovery of the Higgs boson changed physicists' view of the ad hocness of the Higgs mechanism. In contrast, $\mathrm{CCAH}$ accommodates these observations in the following way: (i) and (iv) fall under the heading of " $\mathrm{T}$ provides no reason for believing that $\mathrm{H}^{\prime \prime}$, and (ii) and (iii) are instances where "B provides no reason for believing that $\mathrm{H}^{\prime}$, as our background theories give us no reason why there ought to be only one fundamental scalar particle and why symmetry breaking may proceed differently only in the Higgs mechanism.

In Section 2.2, we mentioned further examples which seem to undermine the independent support view of ad hocness. One may nevertheless wonder whether one could perhaps hold CCAH in conjunction with the independent support condition, in case the independent support condition would get its plausibility from other examples. ${ }^{22}$ First of all, I think it would be important for

\footnotetext{
${ }^{22}$ I owe this objection and one of my replies to the two referees for this journal.
} 
the proponents of the independent support account to provide examples that show that independent support is indeed a concern that scientists have when they deem a hypothesis ad hoc for us to feel forced to consider such a possibility seriously. But we can of course ponder this possibility in the abstract.

On the independent support view of ad hocness, it is sufficient that a hypothesis $\mathrm{H}$ have independent support for it to be non-ad hoc (likewise, a hypothesis must have independent support for it not to be ad hoc). Yet, on my account, what is needed for $\mathrm{H}$ not to be ad hoc is that there are theoretical reasons for belief in $\mathrm{H}$. Independent empirical support is thus not sufficient for non-ad hocness, on my account. In fact, it's not even necessary: even when a hypothesis does not have independent empirical support (but only the evidence it was invoked for), it could count as not ad hoc on my account (namely exactly when there are theoretical reasons for belief in $\mathrm{H}$ ). So my coherentist conception and the independent support notion appear to be incompatible.

Let us now consider the relation of the CCHA to another account of ad hocness. Coherence is intuitively closely related to unfiedness. But although several writers have gestured at a lack of unifiedness when discussing ad hoc features of theories, a precise account of what this would amount to is as of yet wanting. ${ }^{23}$ Suppose the rough idea is that an explanation is unified if it explains a set of phenomena with a small amount of basic assumptions or principles and say that we possessed a particular unified explanation / theory of a certain set of phenomena (Kitcher 1981). Would that mean that none of the phenomena in that set could be accommodated in an ad hoc fashion by the relevant theory? Or

\footnotetext{
${ }^{23}$ I think Leplin's non-fundamentality assumption implicitly appeals to the idea that ad hoc hypotheses are those that are not unified with the theory in question (Leplin 1975). Cf. footnote 3. More recently, Boudry and Leuridan (2011), in their criticism of Sober (2008), seem to be sympathetic to this idea. As mentioned in fn. 16, McMullin associates ad hocness with the absence of coherence, which he seems to think of in terms of lack of unifiedness. Lastly, Lipton may also be ascribed some version of this view. Lipton (1991/2004) has argued for the predictivist thesis that a theory's predictive success should count more than a theory's accommodative success, because there is motivation to "force" the theory to accommodate the evidence and we therefore have reason to suspect that the theory is "fudged" when accommodated. In contrast, scientists generate predictions from their theories "on the basis of the most natural and most explanatory theory" (170-9). Lipton admits that it is a "clear limitation" of his account "that it does not include anything like a precise characterization of the features that make one theoretical system fudgier than another" (180), but does briefly mention that, for him, a fudged theory "becomes more like an arbitrary conjunction, less like a unified theory" (171; added emphasis).
} 
would it mean only at least some of the phenomena would not be accommodated in an ad hoc fashion? The former is implausibly restrictive: many highly unifying theories make ad hoc assumptions (see e.g. the standard model and our discussion of the Higgs mechanism in this paper). But if it's only some phenomena, what is it in the unification account that determines which phenomena are not ad hoc with regard to a unifying theory? In other words, the unification account of ad hocness has an unspecificity problem. This problem is absent on the CCAH. In fact, unification and CCAH are nicely complementary: a unified theory can be coherent to one or another degree, and vice versa, a coherent theory can be unified or not (or maybe unified to one or the other degree). ${ }^{24}$ At the same time, a unified theory will never lack potential coherence, as it presumably will give reasons for believing in at least some of the lower level hypotheses about the phenomena which it unifies (for actual coherence to obtain those reasons must of course be good reasons).

There is another problem of the unification account that the coherence account avoids. As mentioned, unification is usually understood as capturing a number of phenomena with relatively few assumptions. Unification accounts of ad hocness view the need to invoke ad hoc hypotheses for a theory to accommodate the relevant evidence (roughly) as a sign for the lack of unifiedness of a theory. But this idea is not unproblematic. Suppose we have two sets of phenomena P1 and P2 and two theories, A and B, both of which manage to accommodate $\mathrm{P} 1$ and $\mathrm{P} 2$, albeit in different ways. Whereas $\mathrm{A}$ accounts for both P1 and P2 in terms of its own basic assumptions, B accommodates P1 but must to invoke additional hypotheses in order to be able accommodate P2. Intuitively we would want to prefer A over B. Does the unificationist account provide the right resources for justifying this preference? First of all note that $\mathrm{A}$ and $\mathrm{B}$ accommodate the same sets of phenomena. Whether A or B would count as unifying, by the lights of the unificationist account, therefore hinges on the number of principles invoked. Yet, determining this number and whether or not $A$ or $B$ is superior in that regard is by no means a trivial task: as we know from Duhem, both B and A will have to invoke a multitude of auxiliary assumptions in order to be able to accommodate the

\footnotetext{
${ }^{24}$ It has in fact be argued that unification accounts subscribe to a "the winner take all" conception of explanation according to which an explanation either is or is not explanatory, without allowing for degrees of explanation (Woodward 2014).
} 
phenomena, all of which will have to figure in the final count of assumptions. We can therefore not know in advance whether B or (the intuitively preferable) A would come out as more unifying. But this indeterminacy is not tolerable on an account that seeks to provide a principled way of analyzing ad hocness. The proponent of the unificationist account may be able to address this problem by giving us a principled way of distinguishing between fundamental and less fundamental assumptions in a theory, but before this is achieved the coherence account must seem superior to the unificationist account of ad hocness. ${ }^{25}$ To this we may add that the unification account, contrary to CCAH, cannot accommodate the fact that background knowledge seems to play a role in ad hoc judgements, as in the case of the Higgs mechanism (see above).

As mentioned at the very beginning of this essay, some philosophers view ad hocness as the converse of predictive success (Worrall 2002). So, one may ask, how does the CCAH account relate to predictive success, and in particular the thesis that predictive success is better evidence for a theory than accommodative success? On CCAH, there is no asymmetry between evidence that is predicted and evidence that is accommodated. In both cases, CCAH requires good reasons for the hypothesis invoked to predict or accommodate E. So for example, when Einstein, in 1915, was able to derive from the theory of general relativity $(\mathrm{T})$ that light would bend around massive objects such as stars $(\mathrm{H})$, the theory provided reasons for believing in the existence of star light bending, which motivated Eddington and others to go out and collect observational evidence (E) for $\mathrm{H}$. In contrast, the Ptolemaic system, which we briefly considered above, can for example predict that the inner planets will not be observed beyond a certain angle from the sun, but it gives no good reasons for believing in this prediction (because its stipulation of coordination of the position of the sun, Earth, and the centre of an inner planet's epicycle cohere neither with the theory itself or the background knowledge). Thus, although the Ptolemaic system is able to make successful predictions regarding maximum elongation, the basis on which it does this is objectionable. Predictive success therefore does not secure against ad hocness. ${ }^{26}$

\footnotetext{
${ }^{25}$ In fact, Worrall has struggled with this question and concludes that there is no good way of doing so (Worrall 1989, 2002, 2005).

${ }^{26}$ It should be noted that this point does not apply to all accounts of predictive success that can be found in the literature. Although it does apply to the intuitive notion of temporally
} 
Finally, one might perhaps be worried that the CCAH could commit one to a coherentist epistemology for science with all its well-known and highly problematic implications. But that is not the case. Coherentism is the view that a belief is justified if and only if it coheres with other beliefs. Nothing in the CCAH would imply that much. On the contrary, I believe that theories and hypotheses in science are justified when they are supported by the relevant evidence, just as foundationalism has it. Nevertheless those support relations, on CCAH, will be stronger or weaker depending on the amount of coherence between the hypothesis and the theory in question. In other words, the coherence relations modify the support relations. The epistemology to be associated with the CCAH may therefore be referred to as weak foundationalism (Olsson 2012).

\section{The Lorentz-FitzGerald contraction hypothesis revisited}

According to the CCAH, theoretical reasons for belief are crucial for determining whether a hypothesis is ad hoc nor not. In this section I will argue that Lorentz sought to render the contraction hypothesis, a paradigmatic example for an ad hoc hypothesis, non-ad hoc by providing theoretical reasons for belief for it. I take this to confirm the CCAH.

In the analysis of the LFC, one must distinguish between an early and a later, mature, version. The early version was proposed in cursory form by Lorentz in 1895 (and by FitzGerald around the same time) in a short section in the final chapter of a 139-page strong book, the mature version in 1904. Many philosophers of science, for various reasons, believe that the ad hoc charge applies only to the early, but not to the mature version of the LFC (Zahar 1973, Grünbaum 1976, Janssen 2002b, Acuña 2014). This is consistent with scientists such as Einstein deeming the early, but not necessarily the later version of LFC, ad hoc (Holton 1969, 169).

In the early version of LFC, Lorentz assumed that (i) "molecular forces are also transmitted through the ether, like the electric and magnetic forces of

predictive success, it does not apply to others, like Alai's notion of functional novelty, according to which a piece of evidence is novel with regard to a theory $\mathrm{T}$ (which entails $\mathrm{E}$ ) if the use of $\mathrm{E}$ is not essential for the construction of T (Alai 2014). On Alai's account, Ptolemy's predictions would not count as novel, because its predictions are premised on a theory which was constructed under the essential use of the phenomena it predicts. Alai's account bears close resemblance to one version of Worrall's use-novelty account (see ibid. for more details). I thank one of the referees for this journal for pressing me to qualify my claim. 
which we are able at the present time to make this assertion definitely", that (ii) the 'attraction and repulsion' of molecular forces for a body at rest would be in equilibrium, and that (iii) the Lorentz transformations would apply not only to electrostatic forces, but also to the 'molecular forces' holding together matter (Lorentz 1875 in Einstein et al. 1952, 6). As Lorentz readily admitted himself, "there is no reason" in his theory for making the latter assumption in particular. And assumption (ii) is highly implausible (cf. Janssen 2002b, 437).

Some commentators have emphasized that Lorentz's remarks were specifically catered to the Michelson-Morley experiment and that Lorentz articulated the LFC in more general terms only in the later 1904 publication, where he also managed to derive the second order effects he needed to account for the Michelson-Morley experiment (Schaffner 1974, Janssen 2002b). ${ }^{27}$ The mature formulation of the LFC entailed consequences which the early version didn't (or at least not explicitly), such as the velocity-dependence of mass (Janssen 2002b, 425). That consequence was of course testable. It is also for this reason that some philosophers have claimed that the mature LFC was not ad hoc (e.g. Acuña 2014).

But even the mature LFC, it has been argued, is at fault. According to Janssen (2002b):

In Lorentz's theory, there is a strict separation of ether and matter [...] Lorentz decreed a number of important exceptions to the Galilean-invariant Newtonian laws that are supposed to govern matter, so that the laws effectively governing matter are Lorentz invariant. Why, one can legitimately ask, would the laws governing matter have the property of Lorentz invariance, which so far appeared to be nothing but a peculiar property of Maxwell's equations? [...] In the final analysis, it is thus left an unexplained coincidence in Lorentz's theory that both matter and fields are governed by laws that are Lorentz invariant [whereas in Einstein's theory of special relativity, it isn't]. (p. 423 and 426, added emphasis)

Janssen (2002a) cites for approval Poincaré's dismissal of the LFC in his introduction of Sur la dynamique de l'electron (1906):

\footnotetext{
${ }^{27}$ For Janssen $(2002 b, 425)$ the "generalised" LFC is the following: "a matter configuration producing a certain field configuration in a frame at rest in the ether will, when the system is set in motion, change into the matter configuration producing the corresponding state of that field configuration in the frame moving with the system."
} 
We cannot content ourselves with simply juxtaposing formulas that would agree only by some happy coincidence; the formulas should, so to say, penetrate each other.

And indeed, also in the mature theory Lorentz was merely assuming that the Lorentz transformations would apply to 'molecular forces', holding together matter, in the same way they applied to electrostatic forces (Lorentz 1904 in Einstein et al. 1952, 22).

Janssen does not link this perceived deficit of Lorentz's theory to judgments about LFC being ad hoc. Indeed, he concludes that "a solid case can be made for the claim that [Lorentz's mature] theory is not ad hoc by any of the criteria considered here" (ibid., 437). Instead he regards the above shortcoming as a different reason for why Lorentz's theory was inferior to Einstein's. ${ }^{28}$ Yet I don't see why one ought to keep those reasons separate. After all, already in the publication of the early LFC version, as we mentioned before, Lorentz admitted that "there is no reason" to suppose (as he did) that the Lorentz transformations should apply also to matter. But if that's so, then it's not too implausible to suppose that this is also what Einstein and others objected to when deeming the (early) LFC ad hoc.

It is also interesting to note that Lorentz, in a letter to Einstein in 1915, i.e., 10 years after proposing his mature theory, stated his belief that the LFC was rendered non-ad hoc by his offering an explanation for it in terms of molecular forces:

[...] I had added that one can arrive at this hypothesis [i.e., the LFC], if one extrapolates from what one was able to say about the influence of translation on electrostatic forces to other forces. Had I stressed it more, the hypothesis would have made less of an impression of having been devised ad hoc" (Lorentz 1915 in Schulmann et al. 1998, 71-2). ${ }^{29}$

What Lorentz had said in 1875, again, was

that [the LFC] is by no means far-fetched, as soon as we assume that molecular forces are also transmitted through the ether, like electric and magnetic forces of which we are able at present time to make this assertion definitely [...] From the

\footnotetext{
28 See Acuña (2014) for a detailed criticism of Janssen's account.

29 This is my own translation of the original German text.
} 
theoretical side, therefore, there would be no objection to this hypothesis (Lorentz in Einstein et al. 1952, 6, added emphasis).

So what Lorentz appeared to have thought was that the LFC lost its ad hoc character at the moment when he was able to lend to it some theoretical plausibility. And this Lorentz thought despite the fact that he at the same time admitted that he had devised the molecular forces explanation only after he had come up with the LFC (Lorentz 1915 in Schulmann et al. 1998, 74). Although we don't know what Einstein made of that suggestion, ${ }^{30}$ Lorentz doesn't seem to be alone with this judgement. Leplin (1975, fn. 18 p. 314-5) points out, for example, that two later textbooks (one from 1924 and one from 1969) seem to suggest that “Lorentz's representation of contraction as a condition of molecular equilibrium mitigated its ad hoc character" ${ }^{31}$

\subsubsection{Assessment}

From the point of view of CCAH, what Lorentz appeared to have sought to do in order to diminish the ad hoc status of the LFC was to establish a coherence relation between the LFC and the rest of the ether theory. ${ }^{32}$ More specifically, Lorentz sought to offer a causal mechanism for LFC. That he achieved only to a limited degree. Although there perhaps was some plausibility in assuming that the molecular forces that Lorentz postulated for matter would behave not unlike the electromagnetic forces "since both types of force are states of the same substratum", as (Zahar 1973, 116) put it, it remained highly curious how this was to be achieved. As mentioned above, the 'molecular forces' hypothesis required that there be an electrostatic equilibrium when a body is at rest. But there is no such thing as electrostatic equilibrium (cf. Janssen 2002b). Thus, although Lorentz was able to provide some theoretical reasons to believe that LFC, he wasn't able to provide good theoretical reasons. His attempted

\footnotetext{
${ }^{30}$ In his reply to Lorentz, Einstein did not mention the issue (Schulmann et al. 1998).

${ }^{31}$ Zahar (1973) suggests it is for this reason that the LFC is not to be regarded ad hoc. Zahar claims that Lorentz was able to 'derive' the LFC from the molecular force hypothesis. But that's not the case. Lorentz offered only a 'plausibility' argument, no derivation (Janssen 2002b, 436-7). In his mature theory, Lorentz derived the length contraction from what Janssen calls the generalised LFC (see fn. 27, above).

${ }^{32}$ One might be tempted to interpret Lorentz's molecular forces hypothesis as an attempt to produce an explanation that would engender novel predictions and that it was for the latter reason, not for the former, that Lorentz thought the LFC was rendered non-ad hoc. But, as we already noted in Section 2 with Holton, independent predictions of the LFC "were not urged as tests that would decide on its acceptability" (Holton 1969, 177).
} 
explanation of the LFC in terms of molecular forces did not establish coherence with either the ether theory nor with the background theories.

\section{Conclusion}

The historical cases discussed in this paper support a new conception of ad hocness, namely the coherentist conception of ad hocness, which has several conceptual and descriptive advantages over its competitors.

Getting the concept of ad hocness right could have a number of ramifications for other philosophical debates, first and foremost debates concerning theory-confirmation, but also the realism debate. How would current accounts of confirmation be affected by the results of the present study? Could realists exploit the idea that theories which are less ad hoc are better confirmed than theories that possess more ad hoc elements? Answers to these intriguing questions will have to await further research.

\section{References}

Acuña, P. 2014. On the empirical equivalence between special relativity and Lorentz's ether theory. Studies in History and Philosophy of Science Part B: Studies in History and Philosophy of Modern Physics, 46: 283-302.

Alai, M. 2014. Novel predictions and the no miracle argument. Erkenntnis, 79 (2): 297.

Bamford, G. 1993. Popper's Explications of Ad Hocness: Circularity, Empirical Content, and Scientific Practice. The British Journal for the Philosophy of Science, 44 (2): 335355.

- - 1996. Popper and his Commentators on the Discovery of Neptune: A Close Shave for the Law of Gravitation? Studies in History and Philosophy of Science Part A, 27 (2): 207-232.

Barnes, E.C. 2005. On Mendeleev's predictions: comment on Scerri and Worrall. Studies in History and Philosophy of Science Part A, 36 (4): 801-812.

- - 2008. The paradox of predictivism. Cambridge: Cambridge University Press.

BonJour, L. 1985. The structure of empirical knowledge. Cambridge: Cambridge University Press.

Boudry, M. and B. Leuridan. 2011. Where the Design Argument Goes Wrong:

Auxiliary Assumptions and Unification. Philosophy of Science, 78 (4): 558-578.

Carnap, R. 1950. Logical foundations of probability.

Copernicus, N. 1543/1992. On the Revolutions (with a commentary by E. Rosen). Baltimore: Johns Hopkins University Press.

Einstein, A., H. Lorentz, H. Weyl, and H. Minkowski. 1952. The principle of relativity. New York: Dover. 
Feng, J.L. 2013. Naturalness and the Status of Supersymmetry. Annual Review of Nuclear and Particle Science, 63: 351-382.

Friederich, S., R.V. Harlander, and K. Karaca. 2014. Philosophical perspectives on ad hoc hypotheses and the Higgs mechanism. Synthese, 191 (16): 3897-3917.

Gingerich, O. 1975. 'Crisis' versus aesthetic in the Copernican revolution. Vistas in astronomy, 17 (1): 85-95.

Grant, R. 1852. History of physical astronomy. London: Henry G. Bohn.

Grosser, M. 1962. The Discovery of Neptune. Cambridge: Harvard University Press.

Grünbaum, A. 1959. The falsifiability of the Lorentz-Fitzgerald contraction hypothesis. The British journal for the philosophy of science, 10 (37): 48-50.

- - 1976. Ad hoc auxiliary hypotheses and falsificationism. The British Journal for the Philosophy of Science, 27 (4): 329-362.

Heilprin, J. 2013. "Higgs Boson Discovery Confirmed After Physicists Review Large Hadron Collider Data At CERN." Huffington Post, 14/03/13.

http://www.huffingtonpost.com/2013/03/14/higgs-boson-discoveryconfirmed-cern-large-hadron-collider_n_2874975.html.

Holton, G.J. 1969. Einstein, Michelson, and the" crucial" experiment. Isis, 60 (2): 133-197.

- - 1973. Thematic origins of scientific thought: Kepler to Einstein. Harvard: Harvard University Press.

Hunt, J.C. 2012. On Ad Hoc Hypotheses. Philosophy of Science, 79 (1): 1-14.

Janssen, M. 2002a. COI stories: Explanation and evidence in the history of science. Perspectives on Science, 10 (4): 457-522.

- - 2002b. Reconsidering a scientific revolution: The case of Einstein versus Lorentz. Physics in Perspective, 4 (4): 421-446.

Kitcher, P. 1981. Explanatory unification. Philosophy of Science, 48 (4): 507-531.

Krämer, M. 2013. "The landscape of new physics." The Guardian, Janurary 9, 2013.

https://www.theguardian.com/science/life-andphysics/2013/jan/09/physics-particlephysics.

Kuhn, T.S. 1957. The Copernican revolution: planetary astronomy in the development of western thought. Harvard: Harvard University Press.

- - 1977. Objetivity, Value Judgment, and Theory Choice. In The Essential Tension, Chicago: University of Chicago Press, 320-333.

- - 1987. Black-body theory and the quantum discontinuity, 1894-1912. Chicago: University of Chicago Press.

Lakatos, I. 1978. The Methodology of Scientific Research Programmes: Volume 1: Philosophical Papers. Edited by J. Worrall and G. Currie. Cambridge: Cambridge University Press.

Leplin, J. 1975. The concept of an ad hoc hypothesis. Studies In History and Philosophy of Science Part A, 5 (4): 309-345.

- 1982 . The assessment of auxiliary hypotheses. The British Journal for the Philosophy of Science, 33 (3): 235-249.

Lipton, P. 1991/2004. Inference to the best explanation. London: Routledge.

McMullin, E. 1998. Rationality and paradigm change in science (originally appeared in: Horwich, P. (ed.), 1993, World changes: Thomas Kuhn and the nature of 
science, Cambridge: MIT Press). In Philosophy of Science: The Central Issues, Martin Curd and Jan Cover (eds.), New York City: W. W. Norton \& Company, 55-78.

Miller, D. 2014. Representing Space in the Scientific Revolution. Cambridge: Cambridge University Press.

Olsson, E. 2012. Coherentist theories of epistemic justification. The Stanford Encyclopedia of Philosophy (Spring 2013 Edition), edited by Edward N. Zalta, URL = $<$ http://plato.stanford.edu/archives/spr2013/entries/justep-coherence/>.

Overbye, D. 2012. "Physicists Find Elusive Particle Seen as Key to Universe." New York Times, 04/07/2012. http://www.nytimes.com/2012/07/05/science/cernphysicists-may-have-discovered-higgs-bosonparticle.html?_r=2\&pagewanted=all\&.

Popper, K.R. 1959a. The logic of scientific discovery. London: Routledge.

- $1959 \mathrm{~b}$. Testability and 'ad-hocness' of the contraction hypothesis. British Journal for the Philosophy of Science, 10 (37): 50.

Popper, K.R. and P.A. Schilpp. 1974. The Philosophy of Karl Popper: Open Court.

Psillos, S. 1999. Scientific realism: How science tracks truth. London: Routledge.

Scerri, E.R. and J. Worrall. 2001. Prediction and the periodic table. Studies in History and Philosophy of Science Part A, 32 (3): 407-452.

Schaffner, K.F. 1974. Einstein versus Lorentz: research programmes and the logic of comparative theory evaluation. The British Journal for the Philosophy of Science, 25 (1): 45-78.

Schulmann, R., A.J. Kox, M. Janssen, and J. Illy, eds. 1998. The collected papers of Albert Einstein. Vol. 8: The Berlin years: Correspondence 1914-1918. Princeton: Princeton University Press.

Sober, E. 2008. Evidence and evolution: the logic behind the science. Cambridge: Cambridge University Press.

Warburg, E. 1915. Die Physik: Die Kultur der Gegenwart. Leipzig: Teubner.

Weinberg, S. 1967. A model of leptons. Physical Review Letters, 19 (21): 1264.

Wetterich, C. 2012. Where to look for solving the gauge hierarchy problem? Physics Letters B, 718 (2): 573-576.

Woodward, J. 2014. Scientific Explanation. The Stanford Encyclopedia of Philosophy, edited by Edward N. Zalta, http://plato.stanford.edu/archives/win2014/entries/scientificexplanation/.

Worrall, J. 1989. Fresnel, Poisson and the 'White Spot': The Role of Successful Prediction in Theory-acceptance. In The Uses of Experiment, David Gooding, Trevor Pinch and Simon Schaffer (eds.), Cambridge: Cambridge University Press.

- 2002. New evidence for old. In In the Scope of Logic, Methodology and Philosophy of Science, Peter Gardenfors (ed.), Dordrecht: Kluwer, 191-209.

- - 2005. Prediction and the 'periodic law': a rejoinder to Barnes. Studies in History and Philosophy of Science Part A, 36 (4): 817-826. 
Zahar, E. 1973. Why did Einstein's Programme supersede Lorentz's?(I). The British Journal for the Philosophy of Science, 24 (2): 95-123. 